\title{
Acceptability and necessity of HIV and other blood-borne virus testing in a psychiatric setting
}

Camilla Sanger, Janine Hayward, Gira Patel, Karen Phekoo, Alan J. Poots, Cathy Howe, Owen Bowden-Jones and John Green

\begin{abstract}
Summary
Studies in North America and Europe indicate that the prevalence of blood-borne viruses (BBVs) is elevated in individuals with severe mental illness; there are no comparable data for the UK. We offered routine testing for HIV, and hepatitis B and $\mathrm{C}$ in an inner-London in-patient psychiatric unit as a service improvement. Of the patients approached $83 \%$ had mental capacity to provide informed consent for testing and $66 \%$ of patients offered testing
\end{abstract}

accepted. Although it was not our objective to establish the prevalence of BBVs, $18 \%$ of patients had serological evidence of a current or previous BBV infection. We found that offering routine testing in an in-patient psychiatric setting is both practical and acceptable to patients.

\section{Declaration of interest}

None.
In the UK, the majority of HIV testing occurs in sexual health and antenatal services. ${ }^{1}$ Studies outside of the UK have found patients with severe and enduring mental illness to be a high-risk group for blood-borne viruses (BBVs; HIV, hepatitis B and hepatitis C), with reports of HIV seroprevalence of up to $23 \%,{ }^{2}$ hepatitis B up to $25 \%{ }^{3}$ and hepatitis C up to $20 \%{ }^{3,4}$ Individuals with severe mental illness who are sexually active have been found to engage in elevated rates of sexual risk behaviours. ${ }^{2,5,6}$ Routine BBV testing in psychiatric populations is not widespread in the UK, although findings from other countries suggest such a practice would be 'sensible', with mental health services being in a very important position for HIV prevention. ${ }^{7}$ This service improvement project allowed us to assess the acceptability and practicality of routinely offering BBV tests to patients with severe mental illness in a UK acute psychiatric in-patient setting.

\section{Method}

A total of 105 patients of whom $64 \%$ were male aged between 21 and 71 years old from a central London psychiatric hospital (three open admission wards and one intensive care unit) were approached (up to a maximum of three times) and offered BBV tests over a 12-month period. Where patients were identified by staff as very disturbed on a particular day, the offer of a test was delayed and the patient approached later. All participants were provided with an information leaflet and a written account of BBV testing; interpreters were utilised for non-English speakers. Testing was offered by a trained clinical member of the service improvement team who also notified the patient of their results and arranged specialist support for any patient with a positive result. Where possible, tests were undertaken on blood already collected for other tests. Demographic data, psychiatric diagnosis, mental capacity to test, test uptake, test result and transfer to care were collected.

\section{Results}

Table 1 shows that $83 \%(87 / 105)$ of the participants had mental capacity to make an informed decision regarding testing. Of those with mental capacity $66 \%$ (57/87) gave informed consent. During result notification and follow-up there were no reports by patients or staff of patients being distressed by the offer of a test.

Although this was a service improvement project and not an epidemiological study, prevalence of BBVs in this cohort was high.
In total $18 \%(10 / 57)$ of the consenting patients and 13 individuals $(12 \%)$ in the entire cohort showed serological evidence of past or current infection with a BBV or had a known history of BBVs. Four individuals with current or past infections were identified for the first time including one person dually infected with HIV and hepatitis B. A further nine patients were already known to services.

Among the three individuals with HIV one was a new discovery and was referred to specialist support services. Of the participants with hepatitis B serological results showed one had spontaneously cleared the virus and the other three were referred for follow-up (two surface antigen positive and one core antigen positive). All seven individuals with hepatitis $\mathrm{C}$ were already known to services.

\section{Discussion}

Testing for BBVs in a psychiatric setting was acceptable to the majority of patients with severe and enduring mental illness and feasible to deliver. The strategy was successful in identifying and engaging in appropriate care for previously undiagnosed BBVinfected individuals. However, testing was delivered by staff within the service improvement team and further work needs to be conducted to find ways to integrate BBV testing sustainably into standard clinical procedures.

At the outset there were concerns among some staff about patients' capacity to provide informed consent ${ }^{6}$ and about the possibility that offering tests might be disturbing to patients. In practice it was straightforward to obtain consent. Previous research indicates that the vast majority of individuals with schizophrenia are able to provide informed consent. ${ }^{8}$ An educational intervention across more than one session can allow many of those with a reduced capacity to provide informed approval. ${ }^{9}$ Capacity in mental health patients varies over time and some in this cohort who lacked capacity initially were able to consent to BBV testing when re-approached at a later date. Similarly, patients welcomed the offer of a test even when deciding to refuse and reacted with appreciation for the service's interest in their entire well-being rather than appearing distressed.

In conclusion, the routine offering of BBV testing was both acceptable to patients and feasible in this in-patient mental health setting. The project was small and not intended to establish the epidemiology of BBVs among our in-patients and it was carried out in an area where the background population rate of BBVs is high. ${ }^{1}$ However, the prevalence in our cohort was strikingly high, 
Table 1 Acceptability of testing for blood-borne viruses (BBVs; HIV, hepatitis B and hepatitis C) in psychiatric in-patients

\begin{tabular}{|c|c|c|c|c|c|c|c|c|c|c|c|c|}
\hline & \multicolumn{3}{|c|}{$n(\%)$} & \multicolumn{9}{|c|}{$n$} \\
\hline & \multirow{2}{*}{$\begin{array}{l}\text { Approached } \\
\quad(n=105)\end{array}$} & \multirow{2}{*}{$\begin{array}{l}\text { Capacity } \\
(n=87)\end{array}$} & \multirow{2}{*}{$\begin{array}{l}\text { Consent } \\
\text { gained } \\
(n=57)\end{array}$} & \multicolumn{3}{|c|}{$\begin{array}{l}\text { Consenting individuals } \\
\text { with newly identified BBV }\end{array}$} & \multicolumn{3}{|c|}{$\begin{array}{l}\text { Consenting individuals } \\
\text { with known BBV }\end{array}$} & \multicolumn{3}{|c|}{$\begin{array}{l}\text { Non-consenting individuals } \\
\text { with known BBV }\end{array}$} \\
\hline & & & & HIV & Hepatitis B & Hepatitis C & HIV & Hepatitis B & Hepatitis C & HIV & Hepatitis B & Hepatitis C \\
\hline Male & $67(64)$ & $56(64)$ & $41(72)$ & $1^{\mathrm{a}}$ & $3^{a}$ & & 1 & & 4 & & & 2 \\
\hline Female & $38(36)$ & $31(36)$ & $16(28)$ & & 1 & & & & 1 & 1 & & \\
\hline
\end{tabular}

results which are consistent with studies elsewhere suggesting that people with severe mental illness are at increased risk of BBVs. Hepatitis B and C and HIV are treatable conditions; but it is vitally important to diagnose them early. For instance most deaths from HIV occur in those who are detected late, whereas treatment markedly reduces infectiousness and hence, potentially, population spread. There is a strong case for a study to establish the prevalence of BBVs in patients with severe mental illness nationally and for the routine offer of testing to this group nationally, particularly in areas of high-population prevalence. If BBV interventions are to be included as a routine part of patients' care, additional resources and staff training will be required.

Camilla Sanger, BSc Hons, Janine Hayward, MPhil (Cantab), Department of Clinical Health Psychology, Central and North West London NHS Foundation Trust, London; Gira Patel, MBChB, MRCPsych, Department of General Adult Psychiatry, Central and North West London NHS Foundation Trust, London; Karen Phekoo, DMS, DipM, Msoc Sc, PhD, Alan J. Poots, MA(Oxon) MSC, PhD, FLS, Cathy Howe, BSC, DipPsych, MSC, PGC, NIHR Collaboration for Leadership in Applied Health Research and Care for Northwest London, Imperial College London, Chelsea \& Westminster Hospital NHS Foundation Trust, London; Owen Bowden-Jones, MSC MRCPsych, MBChB, Club Drug Clinic, Addictions Directorate, Central and North West London NHS Foundation Trust, London; John Green, PhD, Department of Clinical Health Psychology, Central and North West London NHS Foundation Trust, London, UK

Correspondence: John Green, Department of Clinical Health Psychology, Central and North West London NHS Foundation Trust, 20 Eastbourne Terrace, London W2 6LE, UK. Email: john.green@nhs.net

First received 16 Aug 2012, final revision 25 Jan 2013, accepted 5 Feb 2013

\section{Funding}

Funding was provided by Central and North West London NHS Foundation Trust and the Collaborations for Leadership and Applied Health Research and Care (CLAHRC) programme for North West London.

\section{Acknowledgements}

We thank Dr R Taylor, consultant psychiatrist, and Dr A Mears, consultant physician.

This paper presents independent research by the National Institute for Health Research (NIHR) under the CLAHRC programme for North West London. The views expressed in this publication are those of the authors and not necessarily those of the NHS, NIHR or the Department of Health.

\section{References}

1 Health Protection Agency. HIV in the United Kingdom: 2011 Report. Health Protection Services, 2011.

2 Meade CS, Sikkema KJ. HIV risk behavior among adults with severe mental illness: a systematic review. Clin Psychol Rev 2005; 25: 433-57.

3 Rosenberg SD, Goodman LA, Osher FC, Swartz MS, Essock SM, Butterfield $\mathrm{MI}$, et al. Prevalence of HIV, hepatitis $B$, and hepatitis $C$ in people with severe mental illness. Am J Public Health 2001; 91: 31-7.

4 Meyer JM. Prevalence of hepatitis A, hepatitis B, and HIV among hepatitis C-seropositive state hospital patients: results from Oregon state hospital. J Clin Psychiatry 2003; 64: 540-5.

5 Carey MP, Carey KB, Kalichman SC. Risk for human immunodeficiency virus (HIV) infection among persons with severe mental illness. Clin Psychol Rev 1997; 17: 271-91.

6 Senn TE, Carey MP. HIV testing among individual with a severe mental illness: review, suggestions for research, and clinical implications. Psychol Med 2009; 39: 355-63.

7 Stefan MD, Catalán J. Psychiatric patients and HIV infection: a new population at risk? Br J Psychiatry 1995; 167: 721-7.

8 Moser DJ, Schultz SK, Arndt S, Benjamin ML, Fleming FW, Brems CS, et al. Capacity to provide informed consent for participation in schizophrenia and HIV research. Am J Psychiatry 2002; 159: 1201-7.

9 Carpenter WT, Gold JM, Lahti AC, Queern CA, Conley RR, Bartko JJ, et al. Decisional capacity for informed consent in schizophrenia research. Arch Gen Psychiatry 2000; 57: 533-8. 